\title{
Solution-Processable Graphene Oxide as an Efficient Hole Transport Layer in Polymer Solar Cells
}

\author{
Shao-Sian Li, ${ }^{\dagger, \perp}$ Kun-Hua Tu, ${ }^{\dagger, \perp}$ Chih-Cheng Lin, ${ }^{\dagger}$ Chun-Wei Chen, ${ }^{+, *}$ and Manish Chhowalla ${ }^{\ddagger \S \S}$ \\ ${ }^{\dagger}$ Department of Materials Science and Engineering, National Taiwan University, Taipei 106, Taiwan, ${ }^{\ddagger}$ Department of Materials, Imperial College London, Exhibition Road,

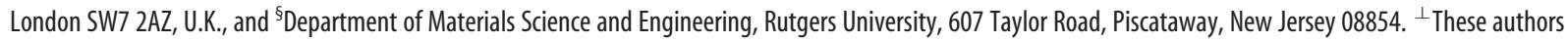 \\ contributed equally to this work.
}

$\mathrm{P}$ olymer solar cells undoubtedly hold tremendous potential for solutionprocessable, inexpensive photovoltaics on flexible plastic substrates. ${ }^{1-5}$ The most promising organic photovoltaics (OPVs) are based on random dispersion of acceptors in the form of organic or inorganic nanostructures within a conjugated polymer matrix, which acts as the donor phase. The interfaces at the acceptor nanostructures and the donor host polymer matrix create bulk heterojunctions (BHJs), which provide a large number of sites for charge separation and bicontinuous pathways for efficient carrier transport. ${ }^{6-8} \mathrm{Al}-$ though numerous combinations of acceptors and donors have been reported, ${ }^{9-14}$ the most popular BHJ polymer solar cells consist of poly(3-hexylthiophene) (P3HT) and fullerene derivative phenyl-C61-butyric acid methyl ester (PCBM) blends. The mechanisms for achieving high photovoltaic efficiencies in excess of $6 \%$ in P3HT:PCBM BHJ devices are well-established. ${ }^{15}$ However, several key components remain unresolved and must be addressed if the theoretical efficiencies of $\sim 10 \%$ are to be realized. ${ }^{16}$ For example, in a simple BHJ device, both the donor and acceptor phases are in direct electrical contact with the cathode and anode electrodes, leading to recombination of carriers and current leakage. To minimize such detrimental effects, electron blocking and hole transport layers (HTLs) are deposited on top of the transparent and conducting indium tin oxide (ITO) anode. HTLs must be wide band gap p-type materials, and several inorganic materials such as $\mathrm{V}_{2} \mathrm{O}_{5}$ and $\mathrm{MoO}_{3}{ }^{17}$ have been reported with $\mathrm{NiO}$ being the most effective, yielding efficiencies greater than $5 \% .{ }^{18}$ However, inorganic HTLs are deposited using vacuum deposition

www.acsnano.org
ABSTRACT The utilization of graphene oxide (GO) thin films as the hole transport and electron blocking layer in organic photovoltaics (OPVs) is demonstrated. The incorporation of $\mathrm{GO}$ deposited from neutral solutions between the photoactive poly(3-hexylthiophene) (P3HT):phenyl-C61-butyric acid methyl ester (PCBM) layer and the transparent and conducting indium tin oxide (ITO) leads to a decrease in recombination of electrons and holes and leakage currents. This results in a dramatic increase in the OPV efficiencies to values that are comparable to devices fabricated with PEDOT:PSS as the hole transport layer. Our results indicate that GO could be a simple solution-processable alternative to PEDOT:PSS as the effective hole transport and electron blocking layer in OPV and light-emitting diode devices.

KEYWORDS: graphene oxide - organic photovoltaics - hole transport layers · organic electronics

techniques that are incompatible with solution-processable printable electronics advantages of OPVs.

Thus, the most commonly employed HTL in polymer solar cells is semiconducting PEDOT:PSS between the ITO anode and the active layer. PEDOT:PSS has the advantages that it is deposited from solution and serves to minimize the detrimental effects of ITO roughness as well as to align the work functions of P3HT and ITO for more efficient collection of holes. However, PEDOT: PSS is usually deposited from highly acidic $(\mathrm{pH} \sim 1)$ aqueous suspensions that are known to corrode ITO at elevated temperatures ${ }^{19}$ and can also introduce water into the active layer, degrading the device performance. ${ }^{20}$ Our research is motivated by the need to overcome the limitations of PEDOT:PSS as the HTL by finding a suitable solution-processable alternative that is compatible with OPV materials and fabrication techniques. Here we demonstrate the utilization of chemically derived graphene oxide (GO) thin films as the HTL in OPVs. The GO thin films are deposited from neutral aqueous suspensions and yield efficiency
*Address correspondence to
chunwei@ntu.edu.tw.

Received for review March 17, 2010 and accepted May 06, 2010.

Published online May 19, 2010. $10.1021 / \mathrm{nn} 100551 \mathrm{j}$

() 2010 American Chemical Society 

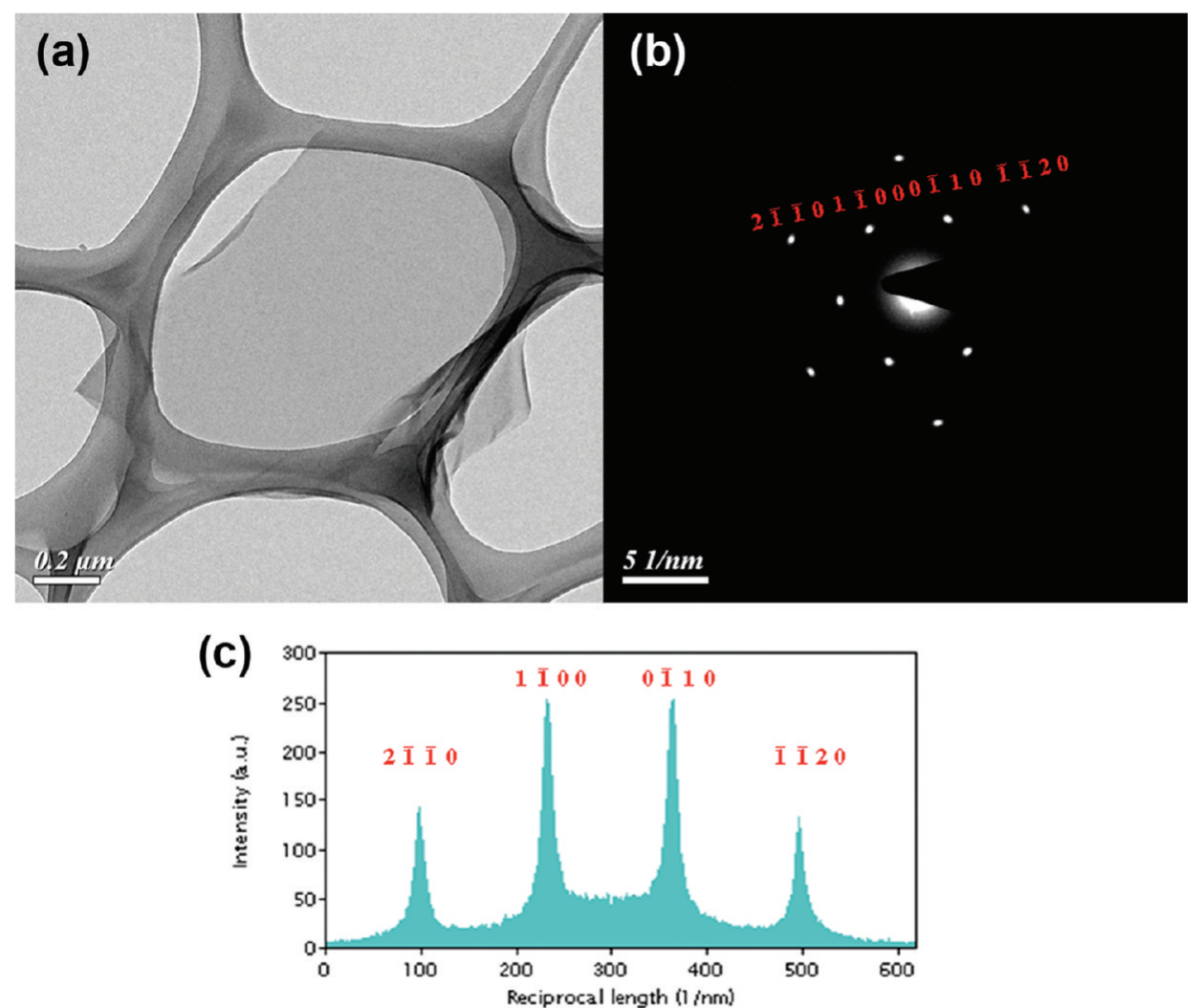

Figure 1. (a) TEM image of as-prepared GO sheet on a lacey carbon support. (b) SAED pattern; the diffraction spots are labeled with Miller-Bravais indices. (c) Relative intensity profile obtained from the diffraction pattern in (b).

values that are comparable to devices fabricated using PEDOT:PSS.

Recently, interest in incorporating graphene into organic photovoltaics has been increasing, particularly as the transparent and conducting electrode. ${ }^{21-24}$ Indeed, good power conversion efficiencies have been obtained from chemical vapor deposited (CVD) graphene as the hole collection electrode. ${ }^{24}$ In addition, the replacement of PCBM with reduced GO has been reported. ${ }^{25-27}$ These results and our present study on using GO as the hole transport layer provide the basis for the development of OPVs based on an all-carbon platform.

Graphene oxide is a graphene sheet functionalized with oxygen groups in the form of epoxy and hydroxyl groups on the basal plane and various other types at the edges. ${ }^{28-30}$ The $\mathrm{C}-\mathrm{O}$ bonds are covalent and thus disrupt the $\mathrm{sp}^{2}$ conjugation of the hexagonal graphene lattice, making GO an insulator. The electronic structure of GO is heterogeneous due to presence of mixed $\mathrm{sp}^{2}$ and $\mathrm{sp}^{3}$ hybridizations and therefore cannot be readily explained by traditional valence and conduction band states. ${ }^{31}$ Rather, lateral transport occurs by hopping between localized states ( $\mathrm{sp}^{2}$ sites) at the Fermi level. ${ }^{32}$ The density of such localized states can be increased by removing oxygen using a variety of chemical and thermal treatments, which facilitate the transport of carriers, ${ }^{33-35}$ making reduced GO a semimetal. ${ }^{32,33}$
In this study, we report the use of nonreduced GO thin films with lateral resistivity values in excess of $10^{5}$ $\Omega / \mathrm{cm}$ as the hole blocking layer in P3HT:PCBM OPVs. It must be clarified that the vertical resistivity of nonreduced GO is an order of magnitude lower $\left(\sim 10^{3}-10^{4}\right.$ $\Omega / \mathrm{cm}$ ) than the lateral resistivity. This is attributed to the fact that the $\mathrm{sp}^{2}$ clusters are isolated laterally but are in contact with the electrodes in a sandwich metal/GO/ metal structure. Thus, carriers (both holes and electrons) can be injected and transported via the isolated $\mathrm{sp}^{2}$ clusters with relative ease in a sandwich device structure. However, because the density of the isolated $\mathrm{sp}^{2}$ clusters is sufficiently low in nonreduced GO, the vertical resistivity is substantial and the conduction and valence bands of the $\mathrm{sp}^{3}$ sites dictate the transport.

$\mathrm{GO}$ was obtained from purified natural graphite powder (SP-1, Bay Carbon) using the modified Hummers method. ${ }^{36} \mathrm{~A}$ concentration of $8 \mathrm{mg} / \mathrm{mL}$ was prepared for deposition of GO thin films. The GO sheets in our groups have been analyzed using numerous analytical techniques and reported in several publications. ${ }^{22,30-32,37-41}$ Here we show typical transmission electron microscopy (TEM) and diffraction results to confirm the structure of GO sheets in as-prepared suspensions. A TEM image of a GO sheet and the corresponding selected area electron diffraction pattern (SAED) are shown in Figure 1a,b and. The 6-fold symmetry in the diffraction pattern is consistent with the hexagonal structure, and the relative intensity of the inner 

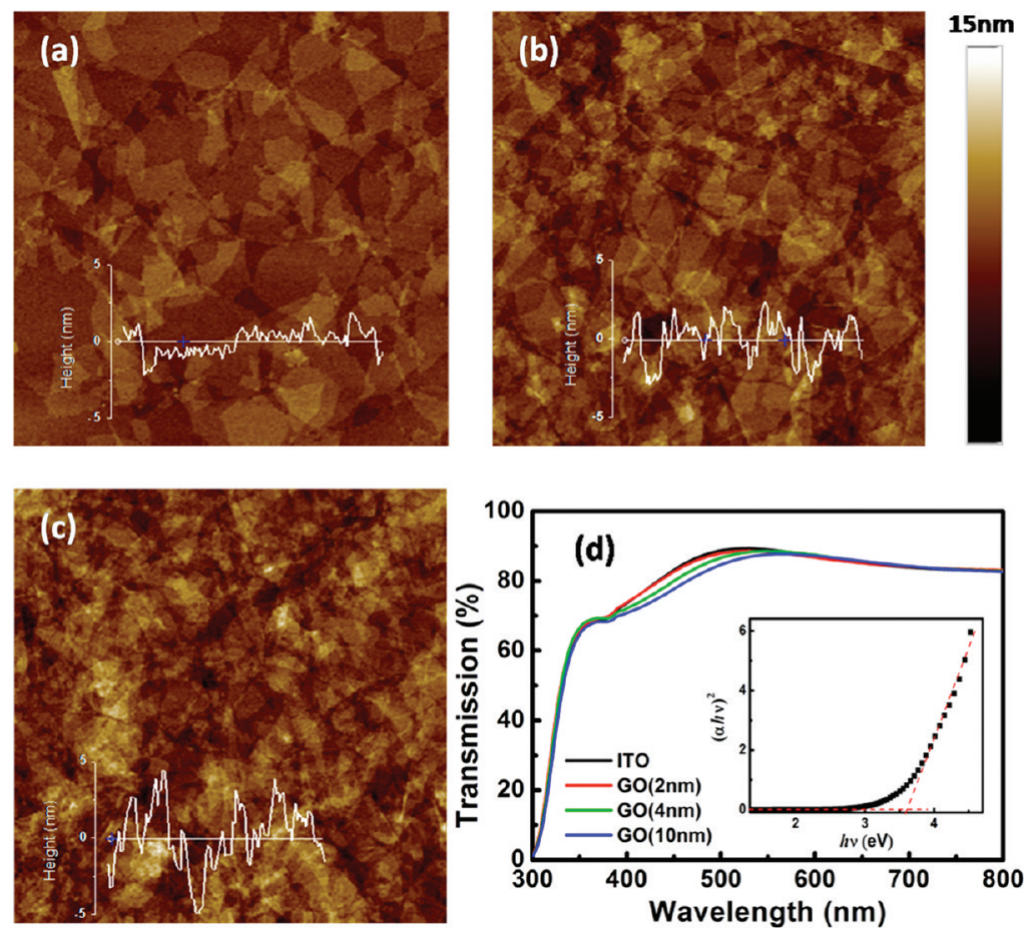

Figure 2. AFM height images of GO thin films with thicknesses of approximately (a) $2 \mathrm{~nm}$, (b) $4 \mathrm{~nm}$, and (c) $10 \mathrm{~nm}$. (d) Corresponding optical transmission spectra of the three GO films deposited on ITO/glass substrates. The inset shows the Tauc plot of the $2 \mathrm{~nm}$ thick GO thin film.

$1 \overline{1} 00$-type and outer $2 \overline{1} \overline{1} 0$-type reflections shown in Figure $1 \mathrm{c}$ are consistent with that of a monolayer. ${ }^{42}$

To investigate the hole transport properties of $\mathrm{GO}$ in OPVs, uniform thin films were deposited on top of ITOcoated glass by spin coating. Atomic force microscope (AFM) topography images of GO thin films of three different thicknesses on ITO are shown in Figure $2 a-c$. The lateral dimensions of GO flakes ranged from 1 to 10 $\mu \mathrm{m}$. GO thin films with three different thicknesses were deposited. The average thicknesses of the three samples were estimated to be $\sim 2, \sim 4$, and $10 \mathrm{~nm}$ with corresponding rms roughness values of $0.70,0.97$, and $1.40 \mathrm{~nm}$, respectively. These values are lower than the 3 $\mathrm{nm}$ rms roughness of bare glass/ITO substrate, indicating that the deposition of GO layers serves to planarize the anode surface. The recent developments in deposition of very uniform thin films with thickness values ranging from a single monolayer to several layers over large areas make it straightforward to incorporate GO as HTL in OPVs. ${ }^{21-24,31-33,41}$ The optical transmission spectra of GO layers with various thicknesses deposited on ITO/glass substrates shown in Figure $2 \mathrm{~d}$ reveal that, although the transmittance decreases slightly with thickness the GO thin films do not significantly alter the transparency of ITO.

As mentioned above, the heterogeneous $\mathrm{sp}^{2} / \mathrm{sp}^{3}$ structure of GO makes it difficult to assign a specific energy for the band gap. Since conduction in GO and reduced GO occurs via tunneling between $\mathrm{sp}^{2}$ sites, $^{31}$ the band gap represents potential barriers for transport formed by covalent $\mathrm{sp}^{3}$ bonds. That is, in as-synthesized www.acsnano.org
$\mathrm{GO}$, the $\mathrm{sp}^{3}$ fraction can be as high as $60 \%$ so that the $\mathrm{sp}^{2}$ domains are nonpercolating, which prevents conduction. ${ }^{31}$ To obtain information about the $\mathrm{sp}^{3}$ potential barriers, the optical gap of GO can be obtained from the Tauc plot $^{43}$ using the relation $\alpha h v \propto\left(h v-E_{\mathrm{g}}\right)^{1 / 2}$, where $\alpha$ is the absorption coefficient and $h v$ is the photon energy, and $E_{\mathrm{g}}$ is the optical gap. The Tauc plot for the $2 \mathrm{~nm}$ thick GO films shown in the inset of Figure $2 \mathrm{~d}$ indicates that $E_{\mathrm{g}}$ is $\sim 3.6 \mathrm{eV}$. This value is comparable to the experimental band gap energy of highly efficient $\mathrm{NiO}$ hole transport layer reported in ref 18.

For the fabrication of OPV devices (area $=0.1 \mathrm{~cm}^{2}$ ), the GO thin films were moved into a nitrogen-purged glovebox for deposition of the organic layer and top electrode. The photoactive layers were deposited on top of the GO thin films by spin coating using a 1:0.8 weight ratio blend of $\mathrm{P} 3 \mathrm{HT}$ :PCBM dissolved in chlorobenzene. The film thickness of the photoactive layer was maintained at $200 \mathrm{~nm}$. Al cathodes were then deposited onto the blend layer by thermal evaporation at a pressure of $2 \times 10^{-6}$ Torr. In this study, we have not utilized LiF layers below the Al cathodes. The photovoltaic device structure consisting of ITO/GO/P3HT: $\mathrm{PCBM} / \mathrm{Al}$ layers is shown in Figure $3 \mathrm{a}$. The band energies of the ITO, P3HT:PCBM, and Al layers are wellknown, while the work function of GO thin films was determined by scanning Kelvin probe microscopy. The average work function values obtained from measurements on 10 different GO thin film samples was found to be $4.9 \mathrm{eV}$, slightly higher than typical values (4.6 eV) obtained for pristine graphene. ${ }^{44}$ The higher values for 

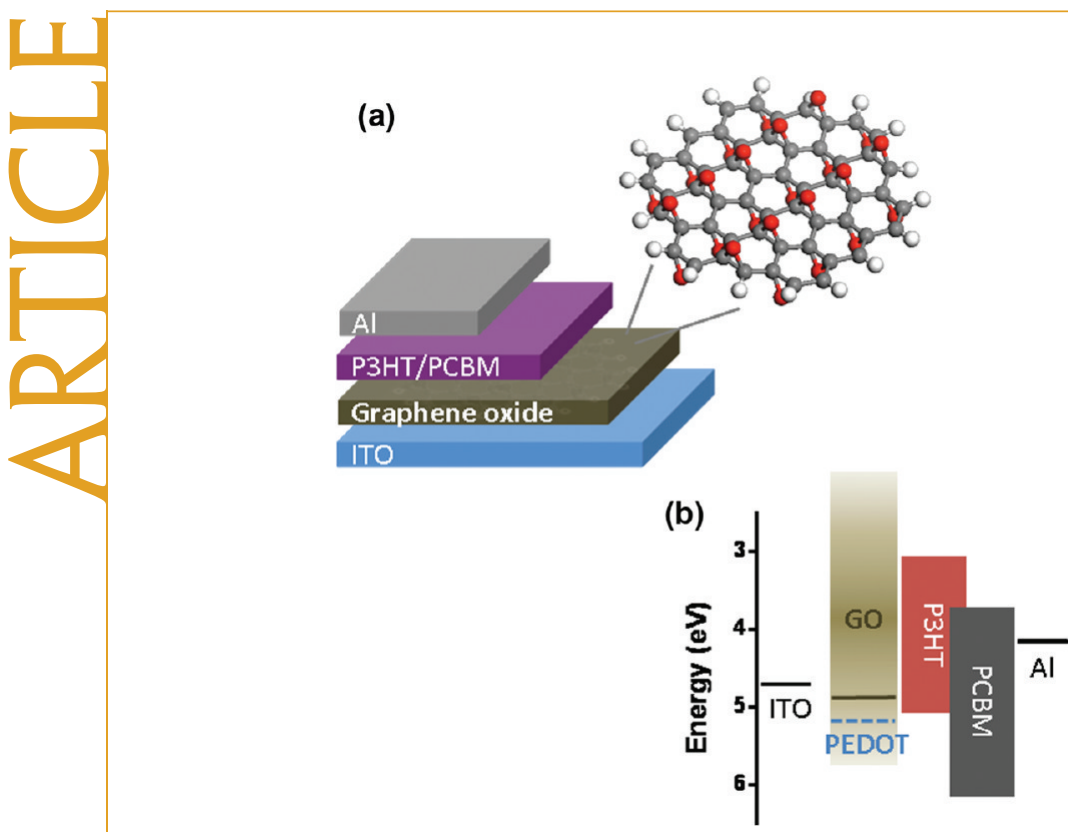

Figure 3. (a) Schematic of the photovoltaic device structure consisting of the following: ITO/GO/P3HT:PCBM/Al. (b) Energy level diagrams of the bottom electrode ITO, interlayer materials (PEDOT:PSS, GO), P3HT (donor), and PCBM (acceptor), and the top electrode Al.

$\mathrm{GO}$ are most likely due to the larger electronegativity of $\mathrm{O}$ atoms, which produce surface $\mathrm{C}^{\delta+}-\mathrm{O}^{\delta-}$ dipoles via extraction of $\pi$ electrons from graphene. ${ }^{45}$ On the basis of the Kelvin probe and optical band gap measurements, the corresponding energy levels of each component are shown in Figure $3 \mathrm{~b}$, although knowledge regarding the position of the Fermi level and exact alignment of energy bands of GO with ITO require further analysis. In addition to GO devices, a set of two other devices was fabricated for comparison. One set was ITO-only control devices consisting of ITO/P3HT: $\mathrm{PCBM} / \mathrm{Al}$ structure, and the other was the conventional OPVs incorporating $30 \mathrm{~nm}$ PEDOT:PSS as the hole transport layer with the following structure: ITO/PEDOT:PSS/ P3HT:PCBM/AI.

The photovoltaic characteristics of the fabricated devices were characterized under simulated A.M. 1.5 illumination at $100 \mathrm{~mW} / \mathrm{cm}^{2}$. The current-voltage plots of the devices are shown in Figure 4a. The average shortcircuit current density $\left(J_{\text {sc }}\right)$, open-circuit voltage $\left(V_{\text {oc }}\right)$, fill factor (FF), and power conversion efficiency values for each set of devices are summarized in Table 1. It can be seen that the ITO-only device exhibits average power conversion efficiency $(\eta)$ of $1.8 \pm 0.2 \%$. The insertion of a $2 \mathrm{~nm}$ thick GO thin film between ITO and P3HT:PCBM results in a substantial increase in $J_{\text {sc, }}, V_{\text {oc }}$ and FF, leading to an enhancement in the power conversion efficiency $(\eta)$ to $3.5 \pm 0.3 \%$. For comparison, typical device performance of ITO/PEDOT:PSS/P3HT: PCBM/AI with efficiency values of around $3.6 \pm 0.2 \%$ is also shown in Figure 4a.

In addition to comparisons with control devices, we have also investigated the influence of GO film thickness on the OPV characteristics, the results of which are
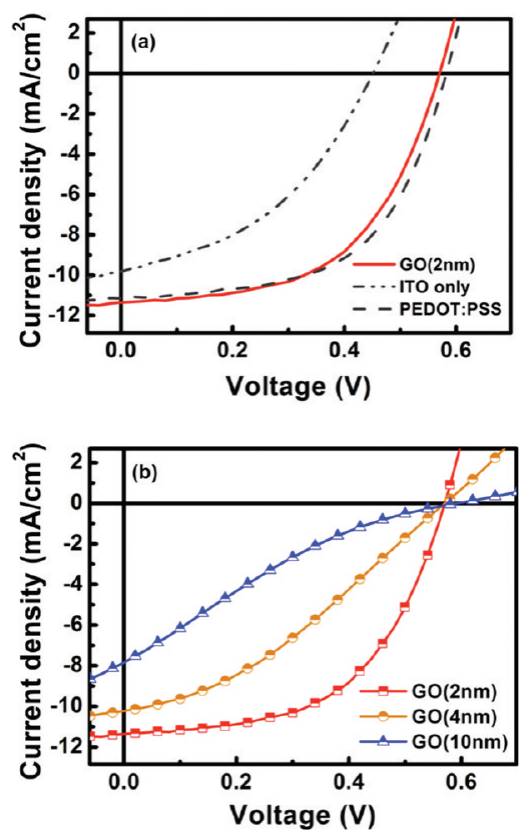

Figure 4. (a) Current-voltage characteristics of photovoltaic devices with no hole transport layer (curve labeled as ITO), with $30 \mathrm{~nm}$ PEDOT:PSS layer, and $2 \mathrm{~nm}$ thick GO film. (b) Current-voltage characteristics of ITO/GO/P3HT: PCBM/Al devices with different GO thickness. All of the measurements were under simulated A.M. 1.5 illumination at $100 \mathrm{~mW} / \mathrm{cm}^{2}$.

shown in Figure 4b. A clear trend of decreasing power conversion efficiency with increasing GO film thickness can be observed. The thinnest film yielded the best results, most likely due to the increase in serial resistance resulting in lower $J_{\mathrm{sc}}$ and FF and the slightly lower transmittance of the films with thickness. The corresponding device characteristics are also summarized in Table 1. The overall efficiencies of devices incorporating GO and PEDOT:PSS are within the measurement errors and also variability in device fabrication procedure. The above results clearly indicate that GO thin films are very promising as the hole transport layers in OPVs.

To obtain insight into the recombination rate and mechanisms in the GO HTL devices, we performed transient open-circuit voltage decay (TOCVD) measurements. Briefly, in TOCVD measurements, devices are illuminated by white light and operated at the steadystate $V_{\text {oc }}$ condition, which can be adjusted by varying the intensity of the bias light. Since no charge is collected at $V_{\text {oc }}$ carriers generated by the short laser pulse have finite lifetimes, $\tau$, before recombining as indi-

TABLE 1. Summary of Typical Photovoltaic Parameters of the Control and GO HTL Devices

\begin{tabular}{lcccc} 
& $\boldsymbol{V}_{\text {oc }}(\mathbf{V})$ & $\boldsymbol{J}_{\text {sc }}\left(\mathbf{m A ~ c m}^{-2}\right)$ & FF (\%) & $\boldsymbol{\eta}(\%)$ \\
\hline ITO only & 0.45 & 9.84 & 41.5 & $1.8 \pm 0.2$ \\
PEDOT:PSS & 0.58 & 11.15 & 56.9 & $3.6 \pm 0.2$ \\
G0 (2 nm) & 0.57 & 11.40 & 54.3 & $3.5 \pm 0.3$ \\
G0 (4 nm) & 0.57 & 10.22 & 33.9 & $2.0 \pm 0.2$ \\
G0 (10 nm) & 0.59 & 7.84 & 18.8 & $0.9 \pm 0.2$
\end{tabular}




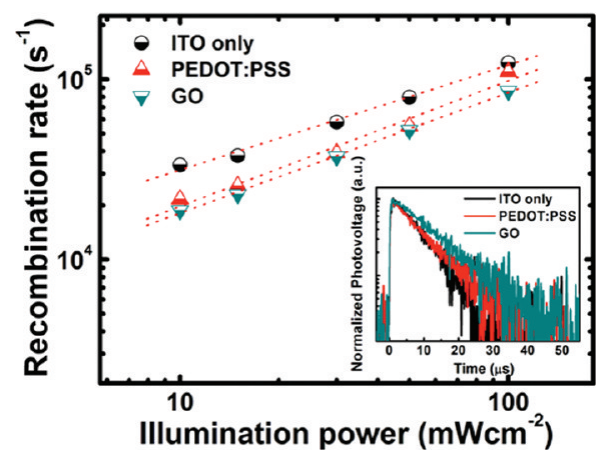

Figure 5. Charge recombination rate constant $k_{\text {rec }}$ versus light intensities obtained using TOCVD measurements for ITO-only, PEDOT:PSS, and $2 \mathrm{~nm}$ GO thin film devices. The inset shows the transient photovoltage decay curves of the corresponding devices measured at $100 \mathrm{~mW} / \mathrm{cm}^{2}$.

cated by the transient decay of the photovoltage. The recombination rate, $k_{\text {rec }}$ is therefore proportional to $1 / \tau$. The charge recombination rate constants versus illumination intensities for the three types of devices tested in this study are shown in Figure 5a. The photovoltage decay curves of the three devices under $100 \mathrm{~mW} / \mathrm{cm}^{2}$ illumination are shown in the inset. The decay lifetimes for ITO-only, PEDOT:PSS HTL, and thin GO HTL devices were found to be 8.1, 9.6, and $11.6 \mu \mathrm{s}$, respectively. The longer recombination lifetimes in $\mathrm{GO}$ indicate lower recombination rates, suggesting effective suppression of leakage current and separation of carriers via efficient transport of holes to ITO and blocking of electrons.

We now briefly consider the hole transport mechanism in GO. From the band diagram (Figure $3 b$ ), it can be seen that the low injection barrier between the GO and $\mathrm{P} 3 \mathrm{HT}$ allows holes to be readily injected into the GO for collection by the ITO electrode. It can be further surmised that the large band gap ( $\sim 3.6 \mathrm{eV})$ of GO hinders transport of electrons from the PCBM LUMO to the ITO anode, acting as an effective electron blocking layer. Unlike PEDOT:PSS, nonreduced GO is insulating (or at least a wide band semiconductor), and in contrast to $\mathrm{NiO}$, where transfer of holes to ITO is facilitated by injection into $\mathrm{Ni}^{2+}$ defect levels close to the valence band, such p-type behavior is not observed in clean GO in our transport measurements. ${ }^{18,31,32}$ The presence of isolated $\mathrm{sp}^{2}$ clusters near the Fermi level should facilitate the transfer of both electrons and holes to ITO and therefore does not contribute to the enhancement in efficiency. The energy level diagram in Figure $3 \mathrm{~b}$ suggests that injection of holes into the valence of $\mathrm{GO}$ is favorable, but the exact configuration of the band alignment requires further investigation.

In summary, we have demonstrated that the thinnest $\mathrm{GO}$ thin films (thickness $=2 \mathrm{~nm}$ ) deposited from neutral solutions are effective hole transport layers in organic photovoltaics. The efficiency values of devices obtained with GO HTLs are comparable to devices fabricated with PEDOT:PSS. Band alignment energies obtained from optical gap and work function measurements of the GO thin films demonstrate that hole transport and electron blocking is facilitated. Further optimization of $\mathrm{GO}$ via the use of nonaqueous samples into OPVs should lead to additional improvements in the device performance as well as the lifetime.

\section{EXPERIMENTAL SETUP}

UV - visible absorption spectra were obtained using a Jasco V-570 UV/vis/NIR spectrophotometer. Current-voltage characteristics (Keithley 2410 source meter) were obtained by using a solar simulator (Newport Inc.) with the A.M. 1.5 filter under irradiation intensity of $100 \mathrm{~mW} / \mathrm{cm}^{2}$. For the transient open-circuit voltage decay (TOCVD) measurements, the devices were measured at open-circuit condition under illumination from the solar simulator with adjustable intensities. A small perturbation generated by a pulse from a frequency-double Nd:YAG pulsed laser $(\lambda=532 \mathrm{~nm}$, repetition rate $=10 \mathrm{~Hz}$, a duration $\sim 5 \mathrm{~ns})$ was used. The transient decay signals were recorded by a digital oscilloscope (Tetronix TDS5052B). The samples were mounted in a vacuum chamber under $10^{-3}$ Torr during measurement. The work function was measured by scanning Kelvin probe microscopy (SKPM) (Innova, Vecco Inc.). For all measurements, Pt/ Ir-coated cantilevers were used.

Acknowledgment. This work is supported by National Science Council, Taiwan (Project No. NSC 98-2119-M-002-020-MY3 and 96-2112-M-002-030-MY3). M.C. would like to acknowledge the support from the Royal Society's Wolfson Merit Award and also the Centre for Advanced Ceramics (CASC) at Imperial College London. The authors would also like to thank Mr. Yen-Fu Chen in Veeco Inc. Asia for the work function measurements.

\section{REFERENCES AND NOTES}

1. Brabec, C. J.; Sariciftci, N. S.; Hummelen, J. C. Plastic Solar Cells. Adv. Funct. Mater. 2001, 11, 15-26.
2. Shaheen, S. E.; Ginley, D. S.; Jabbour, G. E. Organic-Based Photovoltaics. Toward Lowcost Power Generation. MRS Bull. 2005, 30, 10-19.

3. Kim, J. Y.; Kim, S. H.; Lee, H. H.; Lee, K.; Ma, W.; Gong, X.; Heeger, A. J. New Architecture for High-Efficiency Polymer Photovoltaic Cell Using Solution-Based Titanium Oxide as an Optical Spacer. Adv. Mater. 2006, 18, 572-576.

4. Blom, P. W. M.; Mihailetchi, V. D.; Koster, L. J. A.; Markov, D. E. Device Physics of Polymer:Fullerene Bulk Heterojunction Solar Cells. Adv. Mater. 2007, 19, 1551-1566.

5. Li, G.; Shrotriya, V.; Huang, J.; Yao, Y.; Moriarty, T.; Emery, K.; Yang, Y. High-Efficiency Solution Processable Polymer Photovoltaic Cells by Self-Organization of Polymer Blends. Nat. Matter 2005, 4, 864-868.

6. Duren, J. K. J.; Yang, X.; Loos, J.; Bulle-Lieuwma, C. W. T.; Sieval, A. B.; Hummelen, J. C.; Janssen, R. A. J. Relating the Morphology of Poly $(p$-phenylene vinylene)/Methanofullerene Blends to Solar-Cell Performance. Adv. Funct. Mater. 2004, 14, 425-434.

7. Yang, X.; Loos, J.; Veenstra, S. C.; Verhees, W. J. H.; Wienk, M. M.; Kroon, J. M.; Michels, M. A.; Janssen, R. A. J. Nanoscale Morphology of High-Performance Polymer Solar Cells. Nano Lett. 2005, 5, 579-583.

8. Moon, J. S.; Lee, J. K.; Cho, S.; Byun, J.; Heeger, A. J. "Columnlike" Structure of the Cross-Sectional Morphology of Bulk Heterojunction Materials. Nano Lett. 2009, 9, 230-234.

9. Morteani, A. C.; Sreeaunothai, P.; Herz, L. M.; Friend, R. H.; 
Silva, C. Exciton Regeneration at Polymeric Semiconductor Heterojunctions. Phys. Rev. Lett. 2004, 92, 247402.

10. Shaheen, S. E.; Brabec, C. J.; Sariciftci, N. S.; Padinger, F.; Fromherz, T.; Hummelen, J. 2.5\% Efficient Organic Plastic Solar Cells. Appl. Phys. Lett. 2001, 78, 841.

11. Yao, Y.; Shi, C.; Li, G.; Shrotriya, V.; Pei, Q.; Yang, Y. Effects of $\mathrm{C}_{70}$ Derivative in Low Band Gap Polymer Photovoltaic Devices: Spectral Complementation and Morphology Optimization. Appl. Phys. Lett. 2006, 89, 153507.

12. Camaioni, N.; Ridolfi, G.; Casalbore-Miceli, G.; Possamai, G.; Maggini, M. The Effect of a Mild Thermal Treatment on the Performance of a Poly(3-alkykthiophene)/Fullerene Solar Cells. Adv. Mater. 2002, 14, 1735-1738.

13. Soci, C.; Hwang, I.-W.; Moses, D.; Zhu, Z.; Waller, D.; Gaudiana, R.; Brabec, C. J.; Heeger, A. J. Photoconductivity of a Low-Bandgap Conjugated Polymer. Adv. Funct. Mater. 2007, 17, 632-636.

14. Mayer, A. C.; Toney, M. F.; Scully, S. R.; Rivnay, J.; Brabec, C. J.; Scharber, M.; Koppe, M.; Heeney, M.; McCulloch, I.; McGehee, M. D. Bimolecular Crystals of Fullerene in Conjugated Polymers and the Implications of Molecular Mixing for Solar Cells. Adv. Funct. Mater. 2009, 19, 1173-1179.

15. Ma, W.; Yang, C.; Gong, X.; Lee, K.; Heeger, A. J. Thermally Stable, Efficient Polymer Solar Cells with Nanoscale Control of the Interpenetrating Network Morphology. Adv. Funct. Mater. 2005, 15, 1617-1622.

16. Dennler, G.; Scharber, C.; Brabec, C. J. Polymer-Fullerene Bulk Heterojunction Solar Cells. Adv. Mater. 2009, 21, 1323-1338.

17. Shrotriya, V.; Li, G.; Yao, Y.; Chu, C.-W.; Yang, Y. Transition Metal Oxides as the Buffer Layer for Polymer Photovoltaic Cells. Appl. Phys. Lett. 2006, 88, 073508.

18. Irwin, M. D.; Buchholz, D. B.; Hains, A. W.; Chang, R. P. H.; Marks, T. J. p-Type Semiconducting Nickel Oxide as an Efficient-Enhancing Anode Interfacial Layer in Polymer Bulk-Heterojunction Solar Cells. Proc. Natl. Acad. Sci. U.S.A. 2008, 105, 2783-2787.

19. Kim, Y.-H.; Lee, S.-H.; Noh, J.; Han, S.-H. Performance and Stability of Electroluminescent Device with Self-Assembled Layers of Poly(3,4-ethylenedioxythiophene)Poly(styrenesulfonate) and Polyelectrolytes. Thin Solid Films 2006, 510, 305-310.

20. Lagemaat, J.; Barnes, T. M.; Rumbles, G.; Shaheen, S. E.; Coutts, T. J.; Weeks, C.; Levitsky, I.; Peltola, J.; Glatkowski, P. Organic Solar Cells with Carbon Nanotubes Replacing $\ln _{2} \mathrm{O}_{3}: \mathrm{Sn}$ as the Transparent Electrode. Appl. Phys. Lett. 2006, 88, 233503.

21. Tung, V. C.; Chen, L.-M.; Allen, M. J.; Wassei, J. K.; Nelson, K.; Kaner, R. B.; Yang, Y. Low-Temperature Solution Processing of Graphene-Carbon Nanotube Hybrid Materials for High-Performance Transparent Conductors. Nano Lett. 2009, 9, 1949-1955.

22. Eda, G.; Lin, Y.-Y.; Miller, S.; Chen, C.-W.; Su, W.-F.; Chhowalla, M. Transparent and Conducting Electrodes for Organic Electronics from Reduced Graphene Oxide. Appl. Phys. Lett. 2008, 92, 233305.

23. Wu, J.; Becerril, H. A.; Bao, Z.; Liu, Z.; Chen, Y.; Peumans, P. Organic Solar Cells with Solution-Processed Graphene Transparent Electrodes. Appl. Phys. Lett. 2008, 92, 263302.

24. Wang, Y.; Chen, X.; Zhong, Y.; Zhu, F.; Loh, K. P. Large Area, Continuous, Few-Layered Graphene as Anodes in Organic Photovoltaic Devices. Appl. Phys. Lett. 2009, 95, 063302.

25. Liu, Q.; Liu, Z.; Zhang, X.; Yang, L.; Zhang, N.; Pan, G.; Yin, S.; Chen, Y.; Wei, J. Polymer Photovoltaic Cells Based on Solution-Processable Graphene and P3HT. Adv. Funct. Mater. 2009, 19, 894-904.

26. Liu, Z.; Liu, Q.; Huang, Y.; Ma, Y.; Yin, S.; Zhang, X.; Sun, W.; Chen, Y. Organic Photovoltaic Devices Based on a Novel Acceptor Material: Graphene. Adv. Mater. 2008, 20, 3924-3930.

27. Liu, Q.; Liu, Z.; Zhang, X.; Zhang, N.; Yang, L.; Yin, S.; Chen, Y. Organic Photovoltaic Cells Based on an Acceptor of Soluble Graphene. Appl. Phys. Lett. 2008, 92, 223303.
28. Schniepp, H. C.; Li, J.-L.; McAllister, M. J.; Sai, H.; HerreraAlonso, M.; Adamson, D. H.; Prud'homme, R. K.; Car, R.; Saville, D. A.; Aksay, I. A. Functionalized Single Graphene Sheets Derived from Splitting Graphite Oxide. J. Phys. Chem. B 2006, 110, 8535-8539.

29. Lerf, A.; He, H. Y.; Forster, M.; Klinowski, J. Structure of Graphite Oxide Revisited. J. Phys. Chem. B 1998, 102, 44774482.

30. He, H. Y.; Klinowski, J.; Forster, M.; Lerf, A. A New Structural Model for Graphite Oxide. Chem. Phys. Lett. 1998, 287, 53-56.

31. Mattevi, C.; Eda, G.; Agnoli, S.; Miller, S.; Mkhoyan, K. A.; Celik, O.; Mastrogiovanni, D.; Granozzi, G.; Garfunkel, E.; Chhowalla, M. Evolution of Electrical, Chemical, and Structural Properties of Transparent and Conducting Chemically Derived Graphene Thin Films. Adv. Funct. Mater. 2009, 19, 2577-2583.

32. Eda, G.; Mattevi, C.; Yamaguchi, H.; Kim, H.; Chhowalla, M. Insulator to Semimetal Transition in Graphene Oxide. J. Phys. Chem. C 2009, 113, 15768-15771.

33. Eda, G.; Fanchini, G.; Chhowalla, M. Large-Area Ultrathin Films of Reduced Graphene Oxide as a Transparent and Flexible Electronic Material. Nat. Nanotechnol. 2008, 3, 270-274.

34. Gomez-Navarro, C.; Weitz, R. T.; Bittner, A. M.; Scolari, M.; Mews, A.; Burghard, M.; Kern, K. Electronic Transport Properties of Individual Chemically Reduced Graphene Oxide Sheets. Nano Lett. 2007, 7, 3499-3503.

35. Wang, S.; Chia, P.-J.; Chua, L.-L.; Zhao, L.-H.; Png, R.-Q.; Sivaramakrishnan, S.; Zhou, M.; Goh, R. G.-S.; Friend, R. H.; Wee, A. T.-S.; et al. Band-like Transport in SurfaceFunctionalized Highly Solution-Processable Graphene Nanosheets. Adv. Mater. 2008, 20, 3440-3446.

36. Hirata, M.; Gotou, T.; Horiuchi, S.; Fujiwara, M.; Ohba, M. Thin-Film Particles of Graphite Oxide 1: High-Yield Synthesis and Flexibility of the Particles. Carbon 2004, 42, 2929-2937.

37. Eda, G.; Lin, Y.-Y.; Mattevi, C.; Yamaguchi, H.; Chen, H.-A.; Chen, I.-S.; Chen, C.-W.; Chhowalla, M. Blue Photoluminescence from Chemically Derived Graphene. Adv. Mater. 2010, 22, 505-509.

38. Mkhoyan, K. A.; Contryman, A. W.; Silcox, J; Stewart, D. A.; Eda, G.; Mattevi, C.; Miller, S.; Chhowalla, M. Atomic and Electronic Structure of Graphene-Oxide. Nano Lett. 2009, 9, 1058-1063.

39. Eda, G.; Chhowalla, M. Graphene-Based Composite Thin Films for Electronics. Nano Lett. 2009, 9, 814-818.

40. Eda, G.; Unalan, H. E.; Rupesinghe, N.; Amaratunga, G.; Chhowalla, M. Field Emission from Graphene Based Composite Thin Films. Appl. Phys. Lett. 2008, 93, 233502.

41. Yamaguchi, H.; Eda, G.; Mattevi, C.; Kim, H.; Chhowalla, M. Highly Uniform 300 mm Wafer-Scale Deposition of Single and Multilayered Chemically Derived Graphene Thin Films. ACS Nano 2010, 4, 524-528.

42. Meyer, J. C.; Geim, A. K.; Katsnelson, M. I.; Novoselov, K. S.; Obergfell, D.; Roth, S.; Girit, C.; Zettl, A. On The Roughness of Single- and Bi-Layer Graphene Membranes. Solid State Commun. 2007, 143, 101-109.

43. Tauc, J. Optical Properties and Electronic Structure of Amorphous Ge and Si. Mater. Res. Bull. 1968, 3, 37-46.

44. Yu, Y.-J.; Zhao, Y.; Ryu, S.; Brus, L. E.; Kim, K. S.; Kim, P. Tuning the Graphene Work Function by Electric Field Effect. Nano Lett. 2009, 9, 3430-3434.

45. Chen, C.-W.; Lee, M.-H. Ab Initio Calculations of Dimensional and Adsorbate Effects on The Workfunction of Single-Walled Carbon Nanotube. Diamond Relat. Mater. 2003, 12, 565-571. 